\title{
Enhancement of Quantum Tunneling for Excited States in Ferromagnetic Particles
}

\author{
J.-Q. Liang ${ }^{1,2,3}$, Y.-B. Zhang ${ }^{3,2}$, H. J. W. Müller-Kirsten ${ }^{1}$, Jian-Ge Zhou ${ }^{1,4}$, F. \\ Zimmerschied ${ }^{1}$, F.-C. $\mathrm{Pu}^{5,3}$ \\ ${ }^{1}$ Department of Physics, University of Kaiserslautern, 67653 \\ Kaiserslautern, Germany \\ ${ }^{2}$ Institute of Theoretical Physics, Shanxi University, Taiyuan, Shanxi 030006, P. R. China \\ ${ }^{3}$ Institute of Physics and Center for Condensed Matter Physics, Chinese Academy of Sciences, \\ Beijing 100080, P. R. China \\ ${ }^{4}$ Department of Physics, Osaka University, Toyonaka, Osaka 560, Japan \\ ${ }^{5}$ Department of Physics, Guangzhou Normal College, Guangzhou 510400, P. R. China
}

\begin{abstract}
A formula suitable for a quantitative evaluation of the tunneling effect in a ferromagnetic particle is derived with the help of the instanton method. The tunneling between $n$-th degenerate states of neighboring wells is dominated by a periodic pseudoparticle configuration. The low-lying level-splitting previously obtained with the LSZ method in field theory in which the tunneling is viewed as the transition of $n$ bosons induced by the usual(vacuum) instanton is recovered.The observation made with our new result is that the tunneling effect increases at excited states. The results should be useful in analyzing results of experimental tests of macroscopic quantum coherence in ferromagnetic particles.
\end{abstract}




\section{INTRODUCTION}

Macroscopic quantum effects in magnetic systems are of considerable interest both theoretically and experimentally [1]. In the context of these investigations the usual terminology is that macroscopic quantum coherence [2] (MQC) refers to the resonance between neighboring degenerate wells. Some years ago it was reported that MQC was observed for antiferromagnetic particles [3] in resonance experiments. However, the interpretation is controversial [2 [4]. Physically the result of an earlier resonance experiment on ferromagnetic particles is not clear since fundamental discrepancies remain between the experimental data and theoretical expectations on the basis of magnetic quantum tunneling [5,6]. Apart from some other reasons [5],6], which hinder the acceptance of the observation as definite proof of MQC, there is an essential difficulty related to the existing theory of quantum tunneling itself in the absence of an external magnetic field. The difficulty was pointed out in ref. [2]: The argument of the WKB exponential of the tunneling for a ferromagnetic particle is $2 \sqrt{\lambda} s$ with $\lambda=\frac{K_{2}}{K_{1}}, K_{1}$ and $K_{2}$ being the hard and medium axis energies. Since $s \approx 500$ to 5000 , unless $\frac{K_{2}}{K_{1}} \ll 10^{-4}$, the tunneling frequency $\omega_{c}$ is expected to be unobservably small.

It seems that the unobservably small effect of tunneling at the vacuum level is a common phenomenon in various problems, as for instance, in the case of baryon- and lepton-number violation at high energy and in the case of pair production of black holes in quantum gravity. The one loop correction which results in a prefactor of the WKB leading order exponential does not enhance the tunneling significantly in this case. It is a natural and interesting question to ask whether the tunneling effect is enhanced by considering tunneling at the level of excited states. However, the instanton method is suitable only for the evaluation of the tunneling effect at the vacuum level, since the usual(vacuum) instantons satisfy the vacuum boundary conditions. Motivated by the study of baryon- and lepton-number violation at high energy, recently new types of pseudoparticle configurations were found [0 11] which satisfy periodic boundary conditions and are called periodic instantons [8,9] or nonvacuum instantons [10,11]. These periodic instantons have, for instance, been used to evaluate 
quantum tunneling at high energy [12]. There it was confirmed that in the low energy region the tunneling effect indeed increases exponentially with energy [12]. This finding can be expected to have its correspondence in the theoretical analysis of MQC. In the present paper we therefore adopt the periodic instanton method in order to calculate the tunneling amplitude between asymptotically degenerate excited states.

We derive a compact formula for the level-splitting induced by tunneling which is valid for the entire region of energy. The results of the application of a method [13] previously developed for the calculation of tunneling effects at the level of excited states and based on the LSZ (Lehmann, Symanzik and Zimmermann) procedure of field theory [14] are recovered in the low energy region [15]. In particular our formula agrees exactly with the level splitting of the ground state obtained by means of the usual instanton method [16,17]. It is remarkable that the tunneling effect enhances significantly if tunneling at the level of an asymptotically degenerate excited state is considered. We have shown elsewhere that in certain restricted parameter domains the leading contributions of the effect can also be obtained much more easily with Schrödinger quantum mechanics [19], even in the presence of an applied magnetic field [20].

\section{THE EFFECTIVE LAGRANGIAN WITH THE PERIODIC POTENTIAL, AND THE ENERGY SPECTRUM FORMULA}

We begin with the following operator Hamiltonian of the ferromagnetic particle which has been the starting point of numerous investigations

$$
\hat{H}=K_{1} \hat{s}_{z}^{2}+K_{2} \hat{s}_{y}^{2}
$$

and describes [16, 17] XOY easy plane anisotropy and an easy axis along the $\mathrm{x}$ direction with $K_{1}>K_{2}>0$. In eq.(11) $\hat{s}_{i}, i=x, y, z$, are spin operators obeying the usual commutation relation $\left[\hat{s}_{i}, \hat{s}_{i}\right]=i \epsilon_{i j k} \hat{s}_{k}$ (using natural units throughout,i.e. $\hbar=c=1$ ). Starting from the coherent state representation [21] of the time evolution operator with Hamiltonian given by eq.(11) and with the help of the coherent state path-integral we obtain 


$$
\left\langle\mathbf{n}_{f}\left|e^{-2 i \hat{H} T}\right| \mathbf{n}_{i}\right\rangle=e^{-i\left(\phi_{f}-\phi_{i}\right) s} \mathcal{K}\left(\phi_{f}, t_{f} ; \phi_{i}, t_{i}\right)
$$

where

$$
\mathcal{K}\left(\phi_{f}, t_{f} ; \phi_{i}, t_{i}\right)=\int \mathcal{D} \phi \mathcal{D} p e^{i \int_{t_{i}}^{t_{f}} \mathcal{L}(\phi, p) d t}
$$

is the path integral in phase space with canonical variables $\phi$ and $p \equiv s \cos \theta[21]$. Also

$$
\mathcal{L}=\dot{\phi} p-H(\phi, p)
$$

is the phase space (or first order) Lagrangian. The Hamiltonian

$$
H=\frac{p^{2}}{2 m(\phi)}+V(\phi)
$$

has position dependent mass $m(\phi)$ and potential

$$
m(\phi)=\frac{1}{2 K_{1}\left(1-\lambda \sin ^{2} \phi\right)}, \quad V(\phi)=K_{2} s(s+1) \sin ^{2} \phi
$$

respectively where $\lambda \equiv \frac{K_{2}}{K_{1}}$. The kets $\left|\mathbf{n}_{i}\right\rangle$ and $\left|\mathbf{n}_{f}\right\rangle$ denote the initial and final spincoherent states and $t_{f}-t_{i} \equiv 2 T$ denotes the difference of final and initial times. Here $\vec{s}=s(\sin \theta \cos \phi, \sin \theta \sin \phi, \cos \theta)$ is visualized as a classical spin vector with spin number $s$, polar angle $\theta$ and azimuthal angle $\phi$. In the above derivation, the large spin limit $s \gg 1$ has been used since giant spins with spin quantum number $s \gg 1$ are believed to describe ferromagnetic grains. A novel feature of the transition amplitude given by eq.(2) is the phase factor $e^{-i\left(\phi_{f}-\phi_{i}\right) s}$ which can be put into the Lagrangian, i.e. the expression $\phi_{f}-\phi_{i}=\int_{t_{i}}^{t_{f}} \dot{\phi} d t$, and identified as a Wess-Zumino term [22]. Integrating out the momentum in the path integral eq.(3), we obtain the usual Feynman propagator in configuration space, i.e.

$$
\mathcal{K}\left(\phi_{f}, t_{f} ; \phi_{i}, t_{i}\right)=\int \tilde{\mathcal{D}} \phi e^{i \int_{t_{i}}^{t_{f}} \mathcal{L}(\phi, \dot{\phi}) d t}
$$

where $\tilde{\mathcal{D}} \phi$ is the measure-modified functional differential resulting from the $\phi$-dependent mass, i.e.

$$
\tilde{\mathcal{D}} \phi=\prod_{k}^{M-1} \sqrt{\frac{m\left(\phi_{k}\right)}{2 \pi i \epsilon}} d \phi_{k}
$$


with the second order Lagrangian

$$
\mathcal{L}=\frac{1}{2} m(\phi) \dot{\phi}^{2}-V(\phi)
$$

which is more convenient for the instanton method used in the following. The potential $V(\phi)$ is periodic with period $\pi$ (Fig. 1) and there are two minima in the entire region $2 \pi$. We may look at this periodic potential as a superlattice with lattice constant $\pi$ and total length $2 \pi$, and we can derive the energy spectrum in the tight-binding approximation. The translational symmetry is ensured by the possibility of successive $2 \pi$ extensions.

It was shown in a previous paper [23] that if $\epsilon_{m}$ are the degenerate eigenvalues of the system with infinitely high barriers, the energy spectrum is given by

$$
E_{m}=\epsilon_{m}-2 \triangle \epsilon_{m} \cos (s+\xi) \pi
$$

where the expression $\triangle \epsilon_{m}$ is defined by

$$
\triangle \epsilon_{m}=-\int u_{m}^{*}\left(\phi, \Phi_{n}\right) \hat{H} u_{m}\left(\phi, \Phi_{n+1}\right) d \phi
$$

which is the usual overlap integral or $2 \triangle \epsilon_{m}$ simply the level shift due to tunneling through any one of the barriers. Here $u_{m}\left(\phi-\Phi_{n}\right)$ denotes the eigenfunction of the harmonic oscillatorapproximated Hamiltonian $\hat{H}_{0}$ in the $n$-th well, i.e.

$$
H_{0}=\frac{p^{2}}{2 m_{0}}+\frac{1}{2} m_{0} \omega_{0}^{2}\left(\phi-\Phi_{n}\right)^{2}
$$

with $m_{0}=\frac{1}{2 K_{1}}$ and $\omega_{0}^{2}=4 K_{1} K_{2} s(s+1) . \xi$ is an integer and here can assume only either of the two values "0" and " 1 ". For half integer spin $s$ the spectrum eq. (9) is quenched to a single degenerate level with degeneracy two. The quenching is seen to be a consequence of Kramer's theorem which says that for half integer spin $s$ the degeneracy cannot be removed in the presence of the crystal field [23]. 


\section{LEVEL SPLITTING OF GROUND STATE DERIVED WITH THE USUAL INSTANTON METHOD}

Above we recalled the low-lying energy spectrum of a giant spin particle in the large spin limit as obtained in [23]. The energy spectrum eq.(9) leads to the level splitting given by the absolute difference for $\xi=0$ and 1 , namely

$$
\triangle E_{m}=2 \triangle \epsilon_{m}|\cos (s+1) \pi-\cos s \pi|= \begin{cases}4 \triangle \epsilon_{m} & \text { for integer spin } s \\ 0 & \text { for half integer spin } s\end{cases}
$$

The only parameter left unknown is the overlap integral or the level shift $2 \triangle \epsilon_{m}$ which can be evaluated with the help of the instanton method. Instantons in field theory of 1+0 dimensions are viewed as pseudoparticles with trajectories existing in barriers, and are therefore responsible for tunneling. Since instanton trajectories interpolate between degenerate vacua (see Fig. 1 (a)) and satisfy vacuum boundary conditions, the instanton method is only suitable for the calculation of tunneling splitting $4 \triangle \epsilon_{0}$ between neighboring vacua. In the following we first consider tunneling at the vacuum level (i.e. $m=0$ ), which leads to the level shift of the ground state energy, i.e. $2 \triangle \epsilon_{0}$ of eq.(10). Passing to imaginary time by Wick rotation $\tau=i t, \beta=i T$, the amplitude for tunneling from the initial well, say that with $n=0$ (and $\phi_{i}=0$ ), to the neighboring well with $n=1$ (and $\phi_{f}=\pi$ ) and considering large $\beta$, the amplitude for the transition between the corresponding coherent states can be shown to be

$$
\begin{aligned}
<\mathbf{n}(\pi)\left|e^{-2 \beta \hat{H}}\right| \mathbf{n}(0)> & =<\mathbf{n}(\pi)\left|0, \Phi_{1}><0, \Phi_{0}\right| \mathbf{n}(0)>e^{-2 \beta \epsilon_{0}} \sinh \left(2 \beta \triangle \epsilon_{0}\right) \\
& =e^{-i \pi s} \mathcal{K}_{E}\left(\phi_{f}=\pi, \beta ; \phi_{i}=0,-\beta\right)
\end{aligned}
$$

where

$$
\mathcal{K}_{E}=\int \mathcal{D} \phi e^{-S_{E}}
$$

is the Euclidean propagator with Euclidean action defined by

$$
S_{E}=\int_{-\beta}^{\beta} \mathcal{L}_{E} d \tau, \quad \mathcal{L}_{E}=\frac{1}{2} m(\phi) \dot{\phi}^{2}+V(\phi)
$$


A relation similar to eq.(12) applies for a transition between asymptotically degenerate states " $m$ " with $\epsilon_{o}$ replaced by $\epsilon_{m}$. Such transitions will be considered in the next section, and the splitting will similarly be read off from eq.(12). From now on $\dot{\phi}=\frac{d \phi}{d \tau}$ denotes the imaginary time derivative.

In the following the Euclidean propagator $\mathcal{K}_{E}$ is evaluated with the instanton method. After evaluation we compare the result with eq.(14) to find the level shift $2 \triangle \epsilon_{0}$. The instanton configuration which minimizes the Euclidean action $S_{E}$ is

$$
\phi_{c}=\arcsin \left[\cosh ^{2} \omega_{0}\left(\tau-\tau_{0}\right)-\lambda \sinh ^{2} \omega_{0}\left(\tau-\tau_{0}\right)\right]^{-\frac{1}{2}}
$$

with position $\tau_{0}$. The instanton trajectory is shown in Fig. 1 (a) with $\tau_{0}=0$. The Euclidean action evaluated for the instanton trajectory eq.(14), sometimes called the instanton mass, is

$$
S_{c}=\int_{-\infty}^{\infty} m\left(\phi_{c}\right) \dot{\phi}_{c}^{2} d \tau=\sqrt{s(s+1)} \ln \frac{1+\sqrt{\lambda}}{1-\sqrt{\lambda}} \approx\left(s+\frac{1}{2}\right) \ln \frac{1+\sqrt{\lambda}}{1-\sqrt{\lambda}}
$$

in agreement with refs. [16,17] and with refs. [19,20] in the case of small values of $\lambda$. To our understanding it is argued in refs. [16, 17 that except in $e^{-S_{c}}$ one can replace $s(s+1)$ by $s^{2}$, whereas in the exponential factor $\sqrt{s(s+1)}$ has to be approximated by $s+\frac{1}{2}$, even for large $s$. The functional integral $\mathcal{K}_{E}$ can be evaluated with the stationary phase method by expanding $\phi$ about the instanton trajectory $\phi_{c}$ such that $\phi=\phi_{c}+\eta$, where $\eta$ is the small fluctuation with boundary conditions $\eta(\beta)=\eta(-\beta)=0$. Up to the one-loop approximation we have

$$
\mathcal{K}_{E}=e^{-S_{c}} I
$$

where

$$
I=\int_{\eta(-\beta)=0}^{\eta(\beta)=0} \mathcal{D} \eta e^{-\delta S_{E}}
$$

is the fluctuation functional integral with the fluctuation action

$$
\delta S_{E}=\int_{-\beta}^{\beta} \eta \hat{M} \eta d \tau
$$


where

$$
\hat{M}=-\frac{1}{2} \frac{d}{d \tau} m\left(\phi_{c}\right) \frac{d}{d \tau}+\tilde{V}\left(\phi_{c}\right)
$$

with

$$
\tilde{V}\left(\phi_{c}\right)=\frac{1}{2}\left[-m^{\prime}\left(\phi_{c}\right) \ddot{\phi}_{c}-\frac{1}{2} m^{\prime \prime}\left(\phi_{c}\right) \dot{\phi}_{c}^{2}+V^{\prime \prime}\left(\phi_{c}\right)\right]
$$

Here $\hat{M}\left(\phi_{c}\right)$ is the operator of the second variation of the action and $m^{\prime}\left(\phi_{c}\right)=\left.\frac{\partial m(\phi)}{\partial \phi}\right|_{\phi=\phi_{c}}$. As in the usual method of evaluating the fluctuation integral $I$, we expand the fluctuation variable $\eta$ in terms of the eigenmodes of $\hat{M}$ and set $\eta=\Sigma_{n} C_{n} \psi_{n}$, where $\psi_{n}$ denotes the $n$-th eigenfunction of $\hat{M}$, and express the result of the integration as an inverse square root of the determinant of $\hat{M}$. In view of the time translation symmetry of the equation of motion, the functional integral $\mathcal{K}_{E}$ is not well defined when expanded about the classical solutions. The translational symmetry results in zero eigenmodes of the second variation operator $\hat{M}$ of the action (which in the present case, of course, has only one). This problem can be cured by the Faddeev-Popov procedure [24] or in a more systematic way with the help of the BRST transformation [25]. Following the procedure of refs. [23,25] the one instanton contribution to the propagator in the one-loop approximation is calculated to be

$$
\mathcal{K}_{E}^{(1)}=2 \beta \frac{4}{\pi}(1-\lambda)^{-\frac{1}{2}} s^{2} K_{2} e^{-\omega_{0} \beta} e^{-S_{c}}
$$

To obtain the desired result proportional to $\sinh \left(2 \beta \triangle \epsilon_{0}\right)$ in eq.(12), the contributions of the infinite number of instanton and anti-instanton pairs to the one instanton contribution have to be taken into account (the trajectory of one instanton plus a pair is shown in Fig. 1 (b)). Interactions among instantons and anti-instantons are neglected in the dilute instanton-gas approximation. The contribution of one instanton plus $n$ such pairs to the propagator is obtained with the help of the group property of the Feynman path-integral and is found to be

$$
\mathcal{K}_{E}^{(2 n+1)}=\frac{(2 \beta)^{2 n+1}}{(2 n+1) !}\left(\frac{s}{\pi}\right)^{\frac{1}{2}} \lambda^{\frac{1}{4}}\left[2^{2}\left\{\frac{K_{1} K_{2}}{(1-\lambda) \pi}\right\}^{\frac{1}{2}} \lambda^{\frac{1}{4}} s^{\frac{3}{2}}\right]^{2 n+1} e^{-(2 n+1) S_{c}} e^{-\omega_{0} \beta}
$$


Summing over all contributions $\mathcal{K}_{E}^{(2 n+1)}$, the final result of the propagator is found to be

$$
\mathcal{K}_{E}=\lambda^{\frac{1}{4}}\left(\frac{s}{\pi}\right)^{\frac{1}{2}} e^{-\beta \omega_{0}} \sinh \left[2 \beta .2^{2}\left\{\frac{K_{1} K_{2}}{(1-\lambda) \pi}\right\}^{\frac{1}{2}} \lambda^{\frac{1}{4}} s^{\frac{3}{2}} e^{-S_{c}}\right]
$$

Comparing with eq. (12) the level shift is seen to be $2 \triangle \epsilon_{0}$ (note that this is the shift a single level) with

$$
\triangle \epsilon_{0}=2^{2}\left\{\frac{K_{1} K_{2}}{(1-\lambda) \pi}\right\}^{\frac{1}{2}} \lambda^{\frac{1}{4}} s^{\frac{3}{2}} e^{-\left(s+\frac{1}{2}\right) \ln \frac{1+\sqrt{\lambda}}{1-\sqrt{\lambda}}} .
$$

Expanding $\triangle \epsilon_{0}$ in the region of small values of $\lambda$, this is

$$
\triangle \epsilon_{0}=2^{2}\left\{\frac{K_{1} K_{2}}{\pi}\right\}^{\frac{1}{2}} \lambda^{\frac{1}{4}} s^{\frac{3}{2}} e^{-2 s \sqrt{\lambda}}
$$

the level splitting being $4 \triangle \epsilon_{0}$. Since $s$ is a large number (500 to 5000 as cited in the literature [3]), the level shift is suppressed in leading order by the factor

$$
e^{-S_{c}} \simeq e^{-2 s \sqrt{\lambda}}
$$

For $\triangle \epsilon_{0}$ to be observable, plausible values of $\lambda$ are of order $10^{-5}$. Such small values of $\lambda$ may not be realizable in nature. The quantum correction proportional to $e^{\frac{3}{2} \ln s}$ does not increase the tunneling probability significantly.

It may be interesting to compare our level splitting of the ground state $\Delta E_{0}$ with that in refs. [16, 17]. The Hamiltonian of eq.(1) in refs. [16] and [17] equals that of our model if one sets the external field equal to zero $(h=0)$ and makes the replacements $A=K_{2}, B=$ $K_{1}-K_{2}$. By simple algebra one can find that the level splitting eq.(16) in ref. [17] (or eq.(9a) in ref. [16]) coincides exactly with our $\Delta E_{0}=4 \triangle \epsilon_{0}$ which also simplifies to the results of refs. 19,20,23 in the appropriate limits. We can now proceed to consider nonvacuum or periodic instantons which are the pseudoparticles interpolating between asymptotically degenerate excited states, and which reproduce the above result for vacuum instantons in the vacuum limit. 


\section{QUANTUM TUNNELING FOR EXCITED STATES AND THE GENERALIZED FORMULA FOR THE LEVEL SPLITTING}

The periodic instanton method 812 has become a powerful tool for the evaluation of quantum tunneling amplitudes over the entire region of energy. The model at hand can be looked at as one for tunneling at the level of excited states of a sine-Gordon-type potential with a position-dependent mass, which has not been reported previously in the literature. The periodic instanton configuration which minimizes the Euclidean action eq. (13) is seen to satisfy the equation of motion

$$
\frac{m\left(\phi_{p}\right)}{2}\left(\frac{d \phi_{p}}{d \tau}\right)^{2}-V\left(\phi_{p}\right)=-E_{c l}
$$

where $E_{c l}>0$, which is a constant of integration, may be viewed as the classical energy of the pseudoparticle configuration. Through the usual procedure of derivation of the periodic instanton solution, after a laborious but straightforward integration of eq. (27), we obtain the periodic instanton configuration [27]

$$
\phi_{p}=\arcsin \left[\frac{1-k^{2} \operatorname{sn}^{2}(\omega \tau \mid k)}{1-\lambda k^{2} \operatorname{sn}^{2}(\omega \tau \mid k)}\right]^{\frac{1}{2}}
$$

where sn $(\omega \tau \mid k)$ denotes a Jacobian elliptic function of modulus $k$,

$$
k=\sqrt{\frac{n_{1}^{2}-1}{n_{1}^{2}-\lambda}}
$$

with

$$
n_{1}^{2}=\frac{K_{2} s(s+1)}{E_{c l}}, \quad \omega=\omega_{0} \sqrt{1-\frac{\lambda E_{c l}}{K_{2} s(s+1)}}
$$

One can check with $\operatorname{sn}(u \mid 1)=\tanh u$ that for $k^{2}=1$ (corresponding to $E_{c l} \rightarrow 0$ ) the configuration (28) reduces to the instanton of eq.(14). The Jacobian elliptic function sn has real periods $4 n \mathcal{K}(k), n$ being an integer and $\mathcal{K}(k)$ the quarter period given by the usual

elliptic integral of the first kind. The parameter values (29), (30) ensure the periodicity of $\phi_{p}=0$ at $\tau=-2 \beta,+2 \beta$ with a crossover from negative to positive values at $\tau=0$ and 
$\phi=\frac{\pi}{2} \bmod 2 \pi$. Thus the one--way transition from a turning point $a_{1}$ to the other turning point $a_{2}$ is mediated by the instanton-like part or one half of the periodic instanton extending from $\tau=-\beta$ to $\tau=+\beta$ (the periodic instanton itself returning to its original position after time $4 \beta$ ). The trajectory of the periodic instanton eq.(28) is shown schematically in Fig. 1(c) where the trajectory is shifted by an amount $2 \pi$. The instanton-like part starts at time $-\beta$ from turning point $\phi=a_{1}=\arcsin \sqrt{\frac{E_{c l}}{K_{2} s(s+1)}}$ and reaches the other turning point $\phi=a_{2}=\pi-\arcsin \sqrt{\frac{E_{c l}}{K_{2} s(s+1)}}$ at time $\beta$. The Euclidean action of the periodic instanton configuration eq.(28) over the domain from $\tau=-\beta$ to $\tau=+\beta$ can be found to be (cf. refs. 9.10)

$$
S_{p}=\int_{-\beta}^{\beta}\left[m\left(\phi_{p}\right) \dot{\phi}_{p}^{2}+E_{c l}\right] d \tau=W+2 E_{c l} \beta
$$

where

$$
W=\frac{\omega}{\lambda K_{1}}\left[\mathcal{K}(k)-\left(1-\lambda k^{2}\right) \Pi\left(\lambda k^{2}, k\right)\right]
$$

and $\Pi\left(\lambda k^{2}, k\right)$ is the complete elliptic integral of the third kind.

It is now necessary to calculate the amplitude $A^{(1)}$ for the transition mediated by one pseudoparticle configuration - in the present case the instanton-part of the periodic instanton - and then to sum over this contribution with other contributions $A^{(2 n+1)}$ obtained by adding an arbitrary number $(n=1,2,3, \ldots)$ of noninteracting complete periodic instantons (corresponding to addition of instanton-anti-instanton pairs in the dilute gas approximation of the instanton procedure) so that in the path integral representation of the amplitude all possible paths between $-\beta$ and $+\beta$ are taken into account. As is well known, this results effectively in an exponentiation of the first contribution (see e.g. ref. [11]). A calculation very similar to this can be found in reference [10] (cf. there eqs. (4.19), (4.24), (4.25)). We can therefore simply transcribe the result into the present context. The total amplitude corresponding to eq.(12) is then

$$
A=\sum_{m=0}^{\infty} A^{(2 m+1)}=e^{-2 E_{c l} \beta} \sinh \left\{\frac{2 \beta \omega}{4 \mathcal{K}\left(k^{\prime}\right)} e^{-W}\right\}
$$


Comparison with eq. (12) as noted earlier then yields the compact formula $8-10$

$$
\Delta E_{c l}=\frac{\omega}{4 \mathcal{K}\left(k^{\prime}\right)} e^{-W}
$$

$k^{\prime}$ being the complementary modulus of $k$, i.e. $k^{\prime}=\sqrt{1-k^{2}}$. We emphasize that the above formula eq. (33) obtained with the periodic instanton is valid for the entire region of energy $E_{c l}$. The validity of the level splitting of excited states considered previously in the literature [28] was restricted to the quasi uniaxial limit, $1-\lambda \ll 1$. Our approach, however, is valid over the whole range of $\lambda$.

We now consider the low energy limit where $E_{c l}$ is much less than the sphaleron energy (or barrier height $K_{2} s(s+1)$ of the potential), i.e. $k \rightarrow 1, k^{\prime} \rightarrow 0$. Expanding $W$ as power series of $k^{\prime}$ up to quadratic order as in ref. [9] and making use of the oscillator approximation of the periodic potential around one of the minima with the quantization replacement $E_{c l} \rightarrow \epsilon_{m}=\left(m+\frac{1}{2}\right) \omega_{0}$ (as in refs. [8,10]), we then have

$$
W=\left(s+\frac{1}{2}\right) \ln \frac{1+\sqrt{\lambda}}{1-\sqrt{\lambda}}+\left(m+\frac{1}{2}\right) \ln \left[\frac{1-\lambda}{8 \sqrt{\lambda} s}\left(m+\frac{1}{2}\right)\right]-\left(m+\frac{1}{2}\right)
$$

The level shift $2 \Delta \epsilon_{m}$ is finally given with

$$
\Delta \epsilon_{m}=\frac{1}{m !} 2^{3 m+2} \frac{\lambda^{\frac{1}{2}\left(m+\frac{1}{2}\right)} s^{m+\frac{3}{2}}}{(1-\lambda)^{m}}\left\{\frac{K_{1} K_{2}}{\pi(1-\lambda)}\right\}^{\frac{1}{2}} \exp \left[-\left(s+\frac{1}{2}\right) \ln \frac{1+\sqrt{\lambda}}{1-\sqrt{\lambda}}\right]
$$

which reduces to the ground state expression eq.(24) for $m=0$. Eq. (35) provides the linkage from the splitting of excited states (which up to now has been considered only by a perturbative method [26]) to that of the ground state.

It has been pointed out that in the low energy region the level shift $2 \Delta \epsilon_{m}$ may be obtained by an alternative but equally good approach called the LSZ method in which only the vacuum instanton (eq. (14) for the problem at hand) plays a role [12, 13, 15]. The comparison with the LSZ method is explained in the appendix. The level shift formula eq. (35) is suitable for a quantitative evaluation of the tunneling amplitude. We therefore borrow from ref. [5] some data for a ferromagnetic particle in order to demonstrate the difference in $\lambda$ with respect to the result of tunneling at the ground state (but we by no means imply - for the reasons explained in the introduction - that the transition is a result of quantum tunneling). 


\section{Table 1}

Comparison of $\lambda$-values computed for various excited states

\begin{tabular}{|c||c|c|c|}
\hline$m$ & $\lambda$ for $s=3 \times 10^{3}$ & $\lambda$ for $s=2 \times 10^{3}$ & $\lambda$ for $s=10^{3}$ \\
\hline \hline 2 & $0.17350 \times 10^{-4}$ & $0.37641 \times 10^{-4}$ & $1.41226 \times 10^{-4}$ \\
\hline 4 & $0.24543 \times 10^{-4}$ & $0.53458 \times 10^{-4}$ & $2.01990 \times 10^{-4}$ \\
\hline 6 & $0.32066 \times 10^{-4}$ & $0.70031 \times 10^{-4}$ & $2.65869 \times 10^{-4}$ \\
\hline 8 & $0.39985 \times 10^{-4}$ & $0.87500 \times 10^{-4}$ & $3.33361 \times 10^{-4}$ \\
\hline 10 & $0.48316 \times 10^{-4}$ & $1.05899 \times 10^{-4}$ & $4.04587 \times 10^{-4}$ \\
\hline 12 & $0.57064 \times 10^{-4}$ & $1.25235 \times 10^{-4}$ & $4.79555 \times 10^{-4}$ \\
\hline 20 & $0.98155 \times 10^{-4}$ & $3.15062 \times 10^{-4}$ & $20.29119 \times 10^{-4}$ \\
\hline
\end{tabular}

The theoretical level shift calculated with the simple model of the WKB exponential is negligibly small. If the observed frequency reported in ref. [5] were considered to be the result of tunneling from the ground state, $\lambda$ would be $1.01 \times 10^{-5}$. The tunneling effect enhances significantly at excited states. Table 1 shows how $\lambda$ increases with $m$ (the index of excited states) for tunneling frequency $\omega_{c}=500 \mathrm{~Hz}$ which is the level splitting $4 \Delta \epsilon_{m}$ and $s=3 \times 10^{3}, 2 \times 10^{3}$ and $10^{3}$ respectively. For $s=10^{3}$ to attain the frequency $\omega_{c}=500 \mathrm{~Hz}$ the value of $\lambda$ need only be $2.03 \times 10^{-3}$, which may be a physically acceptable quantity for a small ferromagnetic particle.

\section{CONCLUSION}

We have shown that the periodic instanton method as well as the LSZ method are useful for the calculation of tunneling effects at excited states of a spin system at low temperature, the former being valid over the entire region of energy, the latter at low energies. The results are suitable for a quantitative evaluation of the tunneling effect. With the simple model of eq.(1) for a ferromagnetic particle we conclude that the tunneling effect at the level of excited states increases. 


\section{Acknowledgment}

J.-Q.L acknowledges support of the Deutsche Forschungsgemeinschaft and J.-G.Z. support of the A. von Humboldt Foundation and the Japan Society for the Promotion of Science for the award of postdoctoral fellowships. J.-Q.L., Y.-B.Z. and F.-C. P. also acknowledge support of the National Natural Science Foundation of China.

\section{Appendix: Comparison with the LSZ result}

From the viewpoint of the LSZ method the transition amplitude between $m$-th degenerate eigenstates in any two neighboring wells (say those for $n=0,1$ ) is viewed as the transition of $m$ bosons induced by the instanton of eq. (14) and is related with the level shift $2 \Delta \epsilon_{m}$ by

$$
A_{f, i}^{m}=\left\langle m, \Phi_{1}\left|e^{-2 \beta \hat{H}}\right| m, \Phi_{0}\right\rangle=e^{-2 \beta \epsilon_{m}} \sinh 2 \beta \Delta \epsilon_{m}
$$

Following ref. [13 the transition amplitude as well as the S-matrix can be related to the Green's function through the procedure known as the LSZ reduction technique [14]. To this end we construct the interacting Euclidean fields $\phi_{ \pm}$in the classically forbidden region with the help of the instanton configuration of eq.(14). Thus we define

$$
\phi_{+}:=\pi-\phi_{c}, \quad \phi_{-}:=\phi_{c}
$$

such that the interaction fields vanish in their respective asymptotic regions, i.e.

$$
\lim _{\tau \rightarrow \infty} \phi_{+}=0, \lim _{\tau \rightarrow-\infty} \phi_{-}=0
$$

The subscripts "--" and "+" here denote the wells with minima at $\Phi_{0}=0$ and $\Phi_{1}=\pi$

respectively. The Euclidean creation and annihilation operators $\hat{a}_{ \pm}^{\dagger}$ and $\hat{a}_{ \pm}$which create and annihilate an effective boson in wells "+" and "-" respectively are related to the interaction field operators $\hat{\phi}_{ \pm}$by

$$
\begin{aligned}
& \hat{a}_{ \pm}^{\dagger}=\sqrt{\frac{2 m_{0}}{\omega_{0}}} e^{-\omega_{0} \tau} \frac{\stackrel{\leftrightarrow}{\partial}}{\partial \tau} \hat{\phi}_{ \pm}(\tau) \\
& \hat{a}_{ \pm}=-\sqrt{\frac{2 m_{0}}{\omega_{0}}} e^{\omega_{0} \tau} \frac{\stackrel{\leftrightarrow}{\partial}}{\partial \tau} \hat{\phi}_{ \pm}(\tau)
\end{aligned}
$$


where $m_{0}=\frac{1}{2 K_{1}}$, and e.g.

$$
\hat{a}_{+}^{\dagger} \stackrel{\tau \rightarrow \infty}{\rightarrow}-\sqrt{2 m_{0} \omega_{0}}(1-\lambda)^{-\frac{1}{2}}
$$

The transition amplitude of eq.(A.1) can now be written

$$
A_{f, i}^{m}=S_{f, i}^{m} e^{-2 \beta m \omega_{0}}
$$

with S-matrix element

$$
S_{f, i}^{m}=\lim _{\substack{\tau^{i} \rightarrow-\infty \\ \tau^{f} \rightarrow \infty}} \frac{1}{m !}\left\langle 0\left|\hat{a}_{+}\left(\tau_{m}^{f}\right) \ldots \hat{a}_{+}\left(\tau_{1}^{f}\right) \hat{a}_{-}^{\dagger}\left(\tau_{1}^{i}\right) \ldots \hat{a}_{-}^{\dagger}\left(\tau_{m}^{i}\right)\right| 0\right\rangle
$$

The S-matrix element can be evaluated in terms of the Green's function $G$ which arises in its evaluation. Thus

$$
\begin{aligned}
S_{f, i}^{m} & =\lim _{\substack{\tau^{i} \rightarrow-\infty \\
\tau^{f} \rightarrow \infty}} \frac{1}{m !} \prod_{l=1}^{m}\left(-\sqrt{\frac{2 m_{0}}{\omega_{0}}} e^{\omega_{0} \tau_{l}^{f}} \frac{\stackrel{\leftrightarrow}{\partial}}{\partial \tau_{l}^{f}}\right)\left(\sqrt{\frac{2 m_{0}}{\omega_{0}}} e^{-\omega_{0} \tau_{l}^{i}} \frac{\overleftrightarrow{\partial}}{\partial \tau_{l}^{i}}\right) G \\
& =\frac{1}{m !} \prod_{l=1}^{m}\left(\frac{-2 m_{0}}{\omega_{0}}\right) e^{\omega_{0}\left(\tau_{l}^{f}-\tau_{l}^{i}\right)}\left[\frac{\partial^{2} G}{\partial \tau_{l}^{f} \partial \tau_{l}^{i}}+\omega_{0}\left(\frac{\partial G}{\partial \tau_{l}^{f}}-\frac{\partial G}{\partial \tau_{l}^{i}}-\omega_{0} G\right)\right]
\end{aligned}
$$

where the $2 m$-point Green's function is defined as usual, i.e.

$$
G=\left\langle 0\left|\hat{\phi}_{+}\left(\tau_{m}^{f}\right) \ldots \hat{\phi}_{+}\left(\tau_{1}^{f}\right) \hat{\phi}_{-}\left(\tau_{1}^{i}\right) \ldots \hat{\phi}_{-}\left(\tau_{m}^{i}\right)\right| 0\right\rangle
$$

Following the procedure in refs. [12,13,15] the S-matrix element is thus

$$
S_{f, i}^{m}=\frac{1}{m !} \prod_{l=1}^{m}\left\{\left(-\frac{2 m_{0}}{\omega_{0}}\right)\left[\frac{d \phi_{+}\left(\tau_{l}^{f}\right)}{d \tau_{l}^{f}} \frac{d \phi_{-}\left(\tau_{l}^{i}\right)}{d \tau_{l}^{i}}\right]\right\} A_{f, i}^{0}
$$

where

$$
A_{f, i}^{0}=2 \beta 2^{2}\left\{\frac{K_{1} K_{2}}{(1-\lambda) \pi}\right\}^{\frac{1}{2}} \lambda^{\frac{1}{4}} S^{\frac{3}{2}} e^{-S_{c}} e^{-\beta \omega_{0}}
$$

is the amplitude for the transition between degenerate ground states. Using the instanton solution of eq.(14) and taking the large time limit $\tau_{l}^{i} \rightarrow-\infty, \tau_{l}^{f} \rightarrow \infty$ after performing the imaginary time derivatives, we finally obtain the transition amplitude by observing that each pair of vertices in eq. (A.9) contributes a factor $-4 \omega_{0}^{2}$. Then

$$
A_{f, i}^{m}=\frac{1}{m !}\left(\frac{2^{3} m_{0} \omega_{0}}{1-\lambda}\right)^{m} e^{-\omega_{0} m 2 \beta} A_{f, i}^{0}
$$

Comparing eq.(A.11) with eq.(A.1) we recover the level shift $2 \Delta \epsilon_{m}$ of eq.(35) for the lowlying levels. Following refs. [16,17] we here take $\omega_{0}=2 \sqrt{K_{1} K_{2}} s$ for reasons of consistency. 
[1] A. J. Leggett, S. Chakravarty, A. T. Dorsey, M. P. A. Fisher, A. Garg and W. Zwerger, Rev.Mod.Phys.59,1 (1987); Proceedings of the Meeting on Quantum Tunneling of Magnetization - QTM'94, ed. L. Gunther and B. Barbara, NATO ASI Series, Vol. 301 (Kluver, Acad. Publ., 1995).

[2] A. Garg, Phys. Rev. Lett.71, 4249(1993).

[3] D. D. Awschalom, J. F. Smyth, G. Grinstein, D. P. DiVincenzo and D. Loss, Phys. Rev. Lett.68, 3092(1992).

[4] D. D. Awschalom, D. P. DiVincenzo, G. Grinstein and D. Loss, Phys. Rev. Lett.71, 4276(1993).

[5] D. D. Awschalom, M.A. McCord and G. Grinstein, Phys. Rev. Lett. 65, 783(1990).

[6] L. Gunther, Phys. World 3, No.12, 28(1990).

[7] N. S. Manton and T. S. Samols, Phys. Lett. B207, 179(1988); J.-Q. Liang, H. J. W. MüllerKirsten and D. H. Tchrakian, Phys. Lett. B282, 105(1992).

[8] J.-Q. Liang and H. J. W. Müller-Kirsten, Phys. Rev. D46, 4685(1992).

[9] J.-Q. Liang and H.J.W. Müller-Kirsten, Phys. Rev. D50, 6519 (1994).

[10] J.-Q. Liang and H.J. W. Müller-Kirsten, Phys. Rev. D51, 718 (1995).

[11] For a review see J.-Q. Liang and H. J. W. Müller-Kirsten, Topics in Quantum Field Theory - Modern Methods in Fundamental Physics, ed. D. H. Tchrakian (World Scientific, 1996), pp. $54-68$.

[12] J.-Q. Liang and H. J. W. Müller-Kirsten, Phys. Lett. B332, 129(1994).

[13] J.-G. Zhou, J.-Q. Liang, J. Burzlaff, H.J.W. Müller-Kirsten, Phys. Lett. A223, 142 (1996).

[14] See for instance S. S. Schweber, An Introduction to Relativistic Quantum Field Theory (Harper 
and Row, New York, 1961), p.691.

[15] J.-Q. Liang, H. J. W. Müller-Kirsten, J.-G. Zhou, F.-C. Pu, Phys. Lett. A228, 97(1997);in eq. (25) of this paper ' 8 ' has to be replaced by ' 4 ' and in eq. (27) $m_{0}$ by $\frac{m_{0}}{1-\lambda}$.

[16] M. Enz and R. Schilling, J. Phys. C, Solid State Phys. 19, 1765 (1986).

[17] M. Enz and R. Schilling, J. Phys. C, Solid State Phys. 19, L711 (1986).

[18] E. M. Chudnovsky and L. Gunther, Phys. Rev. Lett 60, 661(1988).

[19] J.-Q. Liang, H.J.W. Müller-Kirsten and J.M.S. Rana, Phys. Lett.A (1997), to appear.

[20] J.-Q. Liang, H.J.W. Müller-Kirsten, A.V. Shurgaia and F. Zimmerschied, Kaiserslautern report KL-TH/97/5, unpublished.

[21] A. Perelomov, Generalized Coherent States and Their Applications (Springer, Heidelberg,1986).

[22] E. Fradkin, Field Theories of Condensed Matter Systems(Addison-Wesley, New York, 1991).

[23] J.-Q. Liang, H. J. W. Müller-Kirsten, J.-G. Zhou, Z. Physik B102, 525(1997); note that $\sqrt{2}$ in formula (27) must be removed, the factors ' 3 ' in (30) and (32) are misprints, factors ' 8 ' in (28), (29) must be ' 4 ' and $2 \triangle \epsilon$ in (30), (32) must be $4 \triangle \epsilon$.

[24] L. D. Faddeev and V. N. Popov, Phys. Lett. B25, 29(1967).

[25] J.-G. Zhou, F. Zimmerschied, J.-Q. Liang and H. J. W. Müller-Kirsten, Phys. Lett. B365, 163(1996).

[26] D. A. Garanin, J. Phys. A: Math. Gen. 24, L61(1991).

[27] Y. B. Zhang and J.-Q. Liang (unpublished).

[28] J. L. van Hemmen and A. Sütö, Physica B141, 37(1986). 


\section{Figure Captions}

Fig. 1

The periodic potential and the instanton trajectories:

(a) For one instanton (i.e. vacuum instanton),

(b) For one instanton plus one instanton-anti-instanton pair,

(c) One half of the periodic instanton. 


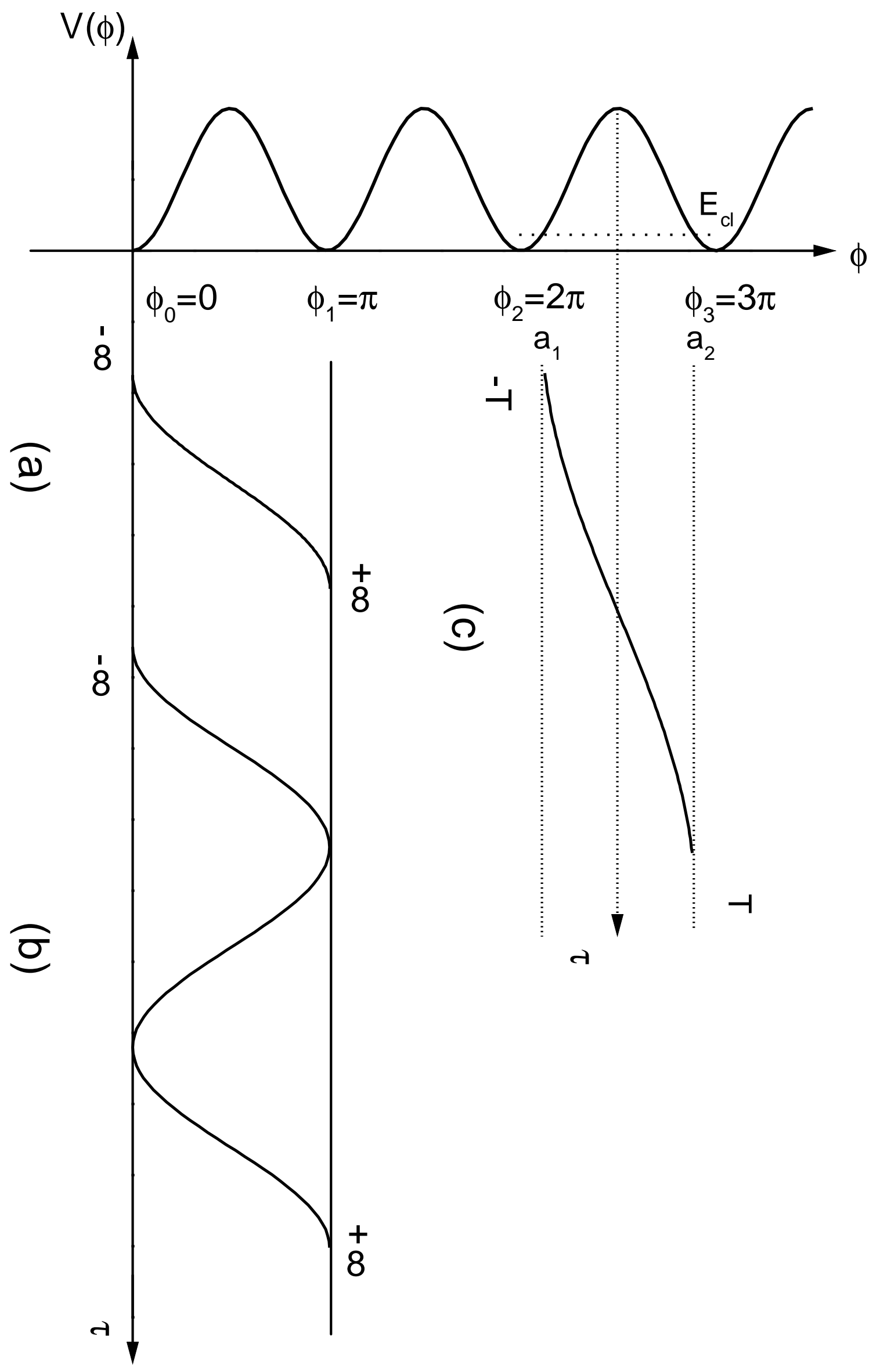

Fig. 1 